\title{
POLARIZATION IMAGING AND SCATTERING MODEL OF CANCEROUS LIVER TISSUES
}

\author{
DONGZHI LI*, ${ }^{*}$, HONGHUI HE*, NAN ZENG*, E DU*, ${ }^{*}$, RAN LIAO*, \\ YONGHONG HE* and HUI MA*,†,* \\ *Shenzhen Key Laboratory for Minimal Invasive Medical Technologies \\ Graduate School at Shenzhen, Tsinghua University \\ Shenzhen 518055, P. R. China \\ ${ }^{\dagger}$ Department of Physics \\ Tsinghua University, Beijing 100084, P. R. China \\ ${ }^{\star}$ mahui@tsinghua.edu.cn \\ SHAOXIONG LIU and MINGHUA LI \\ Shenzhen Sixth People's Hospital (Nanshan Hospital) \\ Huazhong University of Science and Technology \\ Union Shenzhen Hospital, Shenzhen 518052, P. R. China
}

Received 29 May 2013

Accepted 22 June 2013

Published 24 July 2013

\begin{abstract}
We apply different polarization imaging techniques for cancerous liver tissues, and compare the relative contrasts for difference polarization imaging (DPI), degree of polarization imaging (DOPI) and rotating linear polarization imaging (RLPI). Experimental results show that a number of polarization imaging parameters are capable of differentiating cancerous cells in isotropic liver tissues. To analyze the contrast mechanism of the cancer-sensitive polarization imaging parameters, we propose a scattering model containing two types of spherical scatterers and carry on Monte Carlo simulations based on this bi-component model. Both the experimental and Monte Carlo simulated results show that the RLPI technique can provide a good imaging contrast of cancerous tissues. The bi-component scattering model provides a useful tool to analyze the contrast mechanism of polarization imaging of cancerous tissues.
\end{abstract}

Keywords: Polarization imaging; liver cancerous tissues; scattering model; Monte Carlo simulation.

\section{Introduction}

The rate of cancer incidence is currently increasing year by year, and has become a major threat to human health. For both the diagnosis and treatment of cancer, the detection of early-stage cancerous tissues is very essential. ${ }^{1}$ Optical techniques have been widely used in biomedical applications for their characteristics of nondestructive, noncontact and

This is an Open Access article published by World Scientific Publishing Company. It is distributed under the terms of the Creative Commons Attribution 3.0 (CC-BY) License. Further distribution of this work is permitted, provided the original work is properly cited. 
suitable resolution. ${ }^{2,3}$ Meanwhile, the optical properties of pre-cancerous cells are often affected by the physiological and pathological changes of microstructures. $^{1,4,5}$ Thus, the healthy and cancerous tissues can be distinguished by measuring the variations of polarization parameters, which reflect the interactions between polarized photons and microstructures. ${ }^{6-8}$ However, most biological tissues are highly turbid in the visible and near-infrared regions. The "diffusive" photons undergo a large number of scatterings during the imaging process, which reduce the optical contrast, and degrade the result of structural measurements. ${ }^{9}$ Polarization techniques can reduce the impact of the multiple scattered photons to the images, and improve the optical contrast of the superficial tissue imaging. Since more than $85 \%$ of early-stage cancerous changes originate in epidermal tissues, polarization optical methods are of promising prospects for early detection of cancers. ${ }^{10,11}$

In the past decade, several polarization imaging techniques have been developed for biomedical purposes, ${ }^{12}$ particularly for the clinical diagnosis of cancer..$^{9-11,13-15}$ For instance, Anderson et al. reduced the impact of the multiple scattered and surface reflected photons by calculating the difference of two images with perpendicular polarization states [difference polarization imaging (DPI)], and applied it in the diagnosis of skin cancer. ${ }^{13}$ Jacques et al. proposed the degree of polarization imaging method (DOPI), which is the normalized DPI. ${ }^{9}$ They also demonstrated the potential of DOPI in demarcating the margins of cancerous tissues. ${ }^{10,11}$ Recently, some polarimetric methods based on Mueller matrix also have shown the capability for discriminating the normal and carcinoma areas of human tissues. ${ }^{14-16}$ For example, Mueller matrix decomposition has been used effectively to retrieve retardance and depolarization of biological tissues as indicators of cancer detection. ${ }^{17-23}$

In our previous works, we have developed a rotating linear polarization imaging technique (RLPI). It allows us to get a group of new parameters to characterize the microstructural and optical properties of the complicated biological samples. ${ }^{24-27}$ In this paper, we compare imaging contrasts of the cancerous liver tissues using different polarization imaging methods: RLPI, DPI, and DOPI. For a better understanding of the polarization imaging contrast mechanism, we propose a new scattering model for the cancerous liver tissues and carry on a Monte Carlo (MC) simulation. The good agreement between the experiments and MC simulations shows that, a scattering model containing two types of spherical scatterers can explain the contrast relation among the different polarization imaging parameters for the isotropic cancerous liver tissues.

\section{Material and Method}

\subsection{Experimental setup and materials}

The schematic of the RLPI system used in this paper is shown in Fig. 1. The light source is a 1W LED with $650 \mathrm{~nm}$ wavelength. Light from the LED is collimated by a lens L1, and passes through a linear polarizer P1, (Daheng Optics, extinction ratio 500:1). The incident light illuminates the sample at about $25^{\circ}$ to the surface normal direction. ${ }^{9,28}$ The scattered light from the sample passes through another polarizer P2, (Daheng Optics, extinction ratio 500:1) and a lens $\mathrm{L} 2$, and is recorded by a CCD camera (Q-imaging RETIGA EXi). During the measurements, a glass plate is placed in close contact to the sample to avoid surface glare. For RLPI imaging, both $\mathrm{P} 1$ and $\mathrm{P} 2$ are rotated around the optical axis to vary the polarization angles for both illumination $\left(\theta_{i}\right)$ and detection $\left(\theta_{s}\right)$. In this study, the samples are from male nude mice (Experimental Animal Centre of Sun Yat-sen University of Medical Sciences, Guangzhou, China). Tumor samples on the nude mice's livers are obtained by intraperitoneal injection of human colon carcinoma (HCT116) and

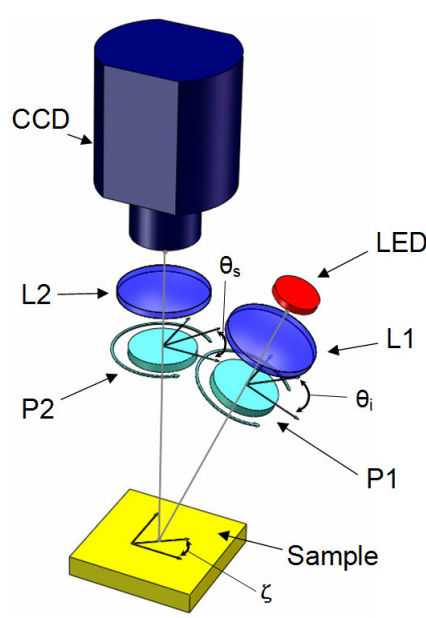

Fig. 1. Schematic of the RLPI system, P: polarizer, L: lens. Both $\mathrm{P} 1$ and $\mathrm{P} 2$ can be rotated around their optical axes to vary the polarization angles for both illumination $\left(\theta_{i}\right)$ and detection $\left(\theta_{s}\right)$. 
nasopharyngeal carcinoma (CNE-1) cells. Both the HCT116 and CNE-1 are commonly used carcinoma models for animals. In the experiments, the carcinoma areas of liver tissues are determined by histological examinations.

\subsection{Parameters of RLPI, DPI and DOPI}

\subsubsection{Difference polarization imaging and Degree of polarization imaging}

DPI technique has been applied to the diagnosis of skin lesions including cancer. ${ }^{13}$ In DPI measurements, samples are illuminated by linearly polarized light, then parallel and perpendicular polarization components of the backscattered light are recorded. The difference of polarization (DP) is defined as Eq. (1), where $\theta_{i}$ represents the angle of linear polarization.

$$
\mathrm{DP}=I_{\|}-I_{\perp}=I\left(\theta_{i}, \theta_{i}\right)-I\left(\theta_{i}, \theta_{i}+\pi / 2\right) .
$$

DOPI has also been applied for cancer detections. ${ }^{9-11}$ The imaging parameter is degree of polarization (DOP, defined as Eq. (2)), which is the DP normalized by the total light intensity to reduce the influence of the illumination light distribution.

$$
\mathrm{DOP}=\frac{I_{\|}-I_{\perp}}{I_{\|}+I_{\perp}}=\frac{I\left(\theta_{i}, \theta_{i}\right)-I\left(\theta_{i}, \theta_{i}+\pi / 2\right)}{I\left(\theta_{i}, \theta_{i}\right)+I\left(\theta_{i}, \theta_{i}+\pi / 2\right)} .
$$

\subsubsection{Rotating linear polarization imaging}

As discussed above, DP and DOP are suitable for the diagnosis of skin cancers. However, we found that when measuring anisotropic biological tissues with linear polarization light, the DP and DOP parameters are closely related to both the incident polarization angle $\left(\theta_{i}\right.$ in Fig. 1) and the orientation of the sample ( $\zeta$ in Fig. 1 ). For the RLPI technique, the parameters are not orientationrelated. ${ }^{24}$ In RLPI measurements, the CCD camera records a series of images corresponding to different combinations of illumination and detection polarization angles $\left(\theta_{i}\right.$ and $\theta_{s}$ in Fig. 1$)$. Then the linear difference polarizations are calculated as Eq. (3):

$$
\operatorname{DP}\left(\theta_{i}, \theta_{s}\right)=I\left(\theta_{i}, \theta_{s}\right)-I\left(\theta_{i}, \theta_{s}+\pi / 2\right) .
$$

The DP images can be fitted to an analytical expression which defines a new set of parameters:

$$
\begin{aligned}
\operatorname{DP}\left(\theta_{i}, \theta_{s}\right)= & \frac{1}{2} I_{i} \sqrt{A \cos \left(4 \theta_{s}-\phi_{1}\right)+B} \\
& \times \cos \left[2 \theta_{i}-\phi_{2}\left(\theta_{s}\right)\right] \\
& +\frac{1}{2} I_{i} C \cos \left(2 \theta_{s}-\phi_{3}\right)
\end{aligned}
$$

$$
\begin{aligned}
A & =\left[B^{2}-(m 22 m 33-m 23 m 32)^{2}\right]^{1 / 2} \\
B & =\left(m 22^{2}+m 23^{3}+m 32^{2}+m 33^{2}\right) / 2 \\
C & =\sqrt{\left(m 21^{2}+m 31^{2}\right)} \\
\tan \varphi_{3} & =m 31 / m 21 .
\end{aligned}
$$

In Eqs. (4) and (5) the fitting parameters A, B, C and $\varphi_{3} / 2$ are independent of the polarization angles of the incident $\left(\theta_{i}\right)$ and scattered $\left(\theta_{s}\right)$ lights. These parameters are related to the structural and optical properties of the sample, such as anisotropy, sizes of the scatterers and alignment of the fibers. ${ }^{24,27}$ The RLPI parameters can be expressed as functions of Mueller matrix elements. We also define $G=A / B$ and prove it correlates to the anisotropy of a sample. ${ }^{24}$

\subsection{Monte Carlo simulation}

For theoretical comparison, we employed a MC simulation program to simulate the propagation and scattering of polarized photon in biological tissues. The MC program is based on a spherecylinder scattering model (SCSM) proposed in our previous work. ${ }^{29-32}$ In SCSM, we apply spherical and cylindrical scatterers to simulate the isotropic and anisotropic structures, which include nuclei and organelles of cells, collagen fibers and muscle fibers. Specially, for some isotropic samples, the cylindrical scatterers can be ignored. Spherical scatterers with different sizes are used to mimic the nuclei and organelles.

In the MC programs, by analytically solving the scalar wave equation, the Mueller matrices of the scatterers are pre-calculated. ${ }^{33}$ Then the MC program launches a normal incident photon with a Stokes vector to represent its polarization state. The photon moves a distance, which is decided by both the scattering and absorption coefficients. At 
each scattering event, a statistical choice is made to decide what type of scatterer the photon hits by considering the probability of scattering for different scatterers. After that, the reference frame of the Stokes vector is rotated by the program, and the phase function is computed by using the pre-calculated scattering matrices. Moreover, the program determines the scattering direction of the photon according to the phase function and a random number. The MC program continues the simulation until either a photon is completely absorbed or moves out of the boundary of the scattering medium. The next photon is then launched. The photons' information, including polarization states, is recorded by the program.

In this paper, the parameters of MC simulations are set to be the same as the experimental tissue samples. The refractive indices of the scatterers and surrounding medium are 1.4 and 1.33 , respectively. ${ }^{34}$ For the simulations of different biological tissues, the diameters of the scatterers, and the scattering coefficients can be set accordingly. Using the scattering models and related MC programs, we can analyze the mechanisms of different polarization imaging techniques for biomedical diagnosis.

\section{Results and Discussions}

\subsection{Polarization imaging for cancerous liver tissues}

DP and DOP have been used for cancer detection. ${ }^{9-11}$ Of particular interest is Human colon carcinoma, as it is one of the most common types of liver metastatic cancer. ${ }^{35}$ We apply RLPI technique to cancerous liver samples, and obtain the DP and DOP parameters from the same measurement. Figure 2 shows the experimental results of the five RLPI parameters ( $\left.\mathrm{G}, \mathrm{A}, \mathrm{B}, \mathrm{C}, \varphi_{3} / 2\right)$, intensity, DP and DOP for a nude mouse's liver, with metastasis of human colon carcinoma (HCT116). In Fig. 2, the carcinoma areas are marked by yellow circle frames, while the other parts are normal liver tissues. The experiments show that polarization imaging can discriminate healthy and cancerous liver tissues. Among the polarization parameters, RLPI parameter B [see Fig. 2(d)] has a higher contrast for distinguishing normal and cancerous tissues of liver samples. Our previous studies have shown that parameters $\mathrm{A}$ and $\mathrm{G}$ are closely related to anisotropy (a)

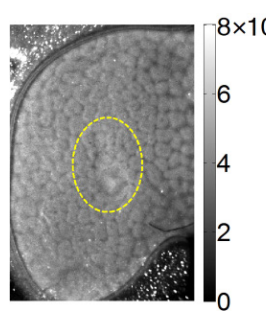

(d)

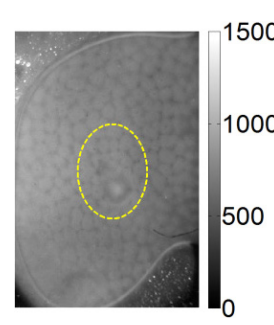

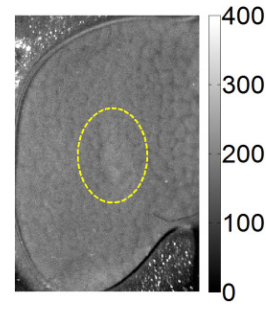

(b)

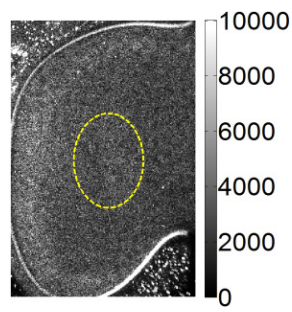

(e)

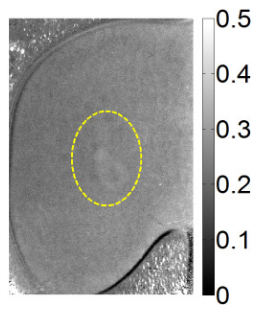

(c)

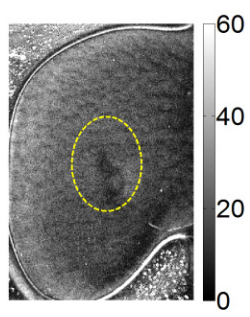

(f)

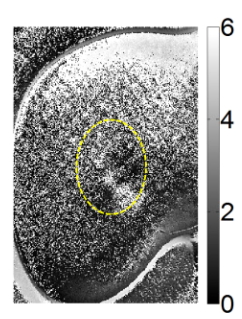

(g)

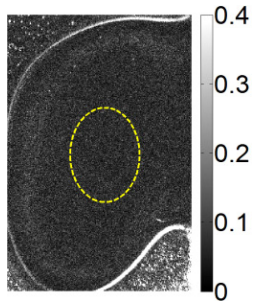

(h)
Fig. 2. Experimental results: (a) intensity, (b) DP, (c) DOP, (d) $\operatorname{RLPI}(\mathrm{B}),(\mathrm{e}) \operatorname{RLPI}(\mathrm{A})$, (f) $\operatorname{RLPI}(\mathrm{C})$, (g) $\operatorname{RLPI}\left(\varphi_{3} / 2\right)$ and (h) RLPI $(\mathrm{G})$ for a nude mouse's liver tissue with human colon carcinoma HCT116. The carcinoma areas are marked by yellow circle frames.

structures. ${ }^{24} \mathrm{G}$ is close to unity for tissues containing well-aligned fibers, but close to zero for isotropic tissues. In Fig. 2, both A and G are very small indicating that the cancerous liver tissues are highly isotropic.

Besides the sample with metastasis of human colon carcinoma, we also conducted polarization measurements on other types of liver cancers. Figure 3 shows the experimental results for a nude mouse's liver with metastasis of nasopharyngeal carcinoma (CNE-1), and the carcinoma areas are marked by yellow circle frames. The results show that $B$ is very sensitive to the existence of cancerous cells. A and $\mathrm{G}$ are also close to zero confirming that the liver tissues are highly isotropic. We select two areas from healthy (marked as green squares) and cancerous liver tissue (marked as red squares), and calculate the imaging contrasts for intensity, DP, 


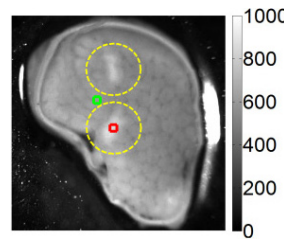

(a)

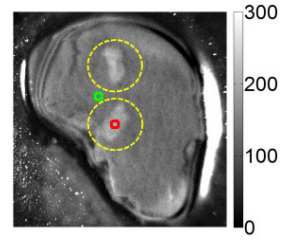

(b)

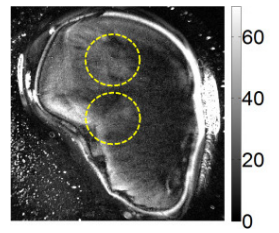

(f)

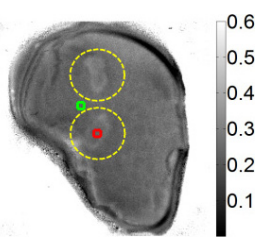

(c)

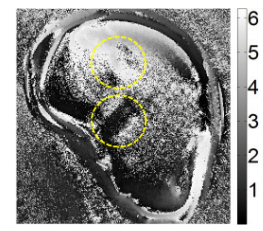

(g)

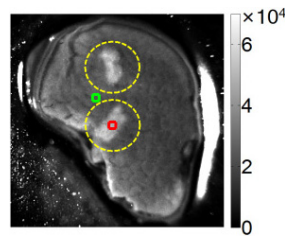

(d)

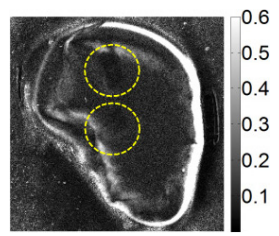

(h)

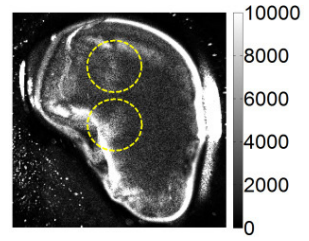

(e)

Fig. 3. Experimental results: (a) intensity, (b) DP, (c) DOP, (d) RLPI(B), (e) RLPI(A), (f) RLPI(C), (g) RLPI $\left(\varphi_{3} / 2\right)$ and (h) RLPI $(\mathrm{G})$, for a nude mouse's liver with metastasis of nasopharyngeal carcinoma CNE-1. The carcinoma areas are marked by yellow circle frames. Contrast between the healthy (marked as green squares) and cancerous liver tissue (marked as red squares) for intensity, DP, DOP and B are: 0.21, 0.17, 0.03 and 0.34 .

DOP and RLPI parameter B. As shown in Fig. 3, the contrast of different parameters are: 0.21 for intensity, 0.17 for DP, 0.03 for DOP and 0.34 for B. The B parameter [see Fig. 3(d)] gives the highest contrast among all the parameters. In Fig. 3(d), the margin between the healthy and cancerous areas is also clearer compared to other three parameters.

\subsection{Scattering model and $M C$ simulation for cancerous liver tissues}

In order to analyze the contract mechanism of polarization measurements, we use scattering models and MC simulations to reproduce the experimental results. Since both the healthy and carcinoma liver tissues are highly isotropic, we firstly use a model containing single type of spherical scatterer to simulate the experimental results shown in Fig. 3. The simulated imaging contrasts of intensity, DP, DOP and RLPI parameter B are shown in Fig. 4. The parameters in $\mathrm{MC}$ simulations are set according to the liver tissues: the diameter and refractive index of spherical scatterer are $7 \mu \mathrm{m}$ (representing the cell nuclei) and 1.4, respectively. The scattering coefficient varies from $70 \mathrm{~cm}^{-1}$ to $270 \mathrm{~cm}^{-1}$ to simulate the cancer-induced cell proliferation. ${ }^{36}$ It can be observed in Fig. 4 that as the concentration for spherical scatterers increases, the relative contrasts of all the four parameters become larger. However, the relation of the four contrasts in descending order is: intensity, $\mathrm{B}, \mathrm{DOP}$ and DP, which is different from the experimental results shown in Fig. 3. During the MC simulation process, the structural parameters including sizes and scattering coefficients are adjusted, and we find that the single-component scattering model cannot generate results matching the experiments.

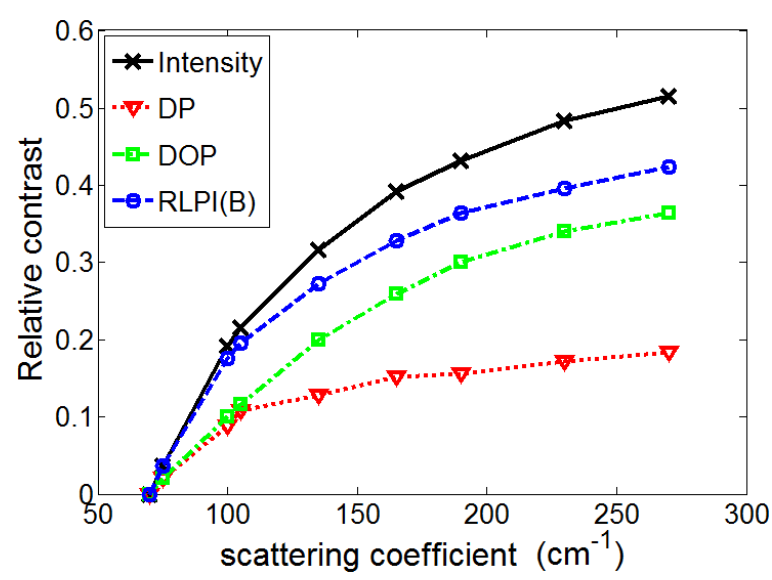

Fig. 4. Monte Carlo simulated relative contrasts of four imaging parameters: intensity, DP, DOP, RLPI parameter B, at different scattering coefficients for a monodisperse sphere scattering model. The diameter and refractive index of spherical scatterer are $7 \mu \mathrm{m}$ and 1.4 , respectively. The scattering coefficient varies from $70 \mathrm{~cm}^{-1}$ to $270 \mathrm{~cm}^{-1}$ to simulate the cancerinduced cell proliferation. 


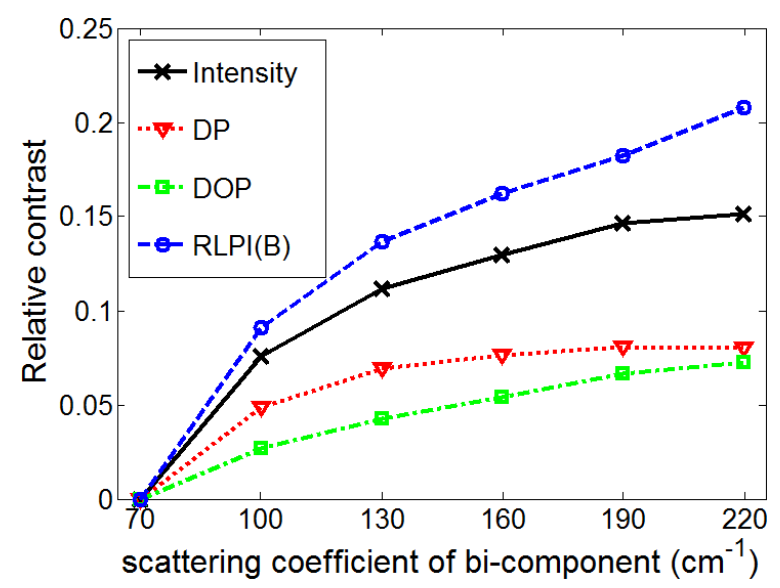

Fig. 5. Monte Carlo simulated relative contrasts of four imaging methods: intensity, DP, DOP, RLPI parameter $\mathrm{B}$, at different concentrations for a scattering model with two components of spherical scatterers. The diameters of small and large spherical scatterer are $0.7 \mu \mathrm{m}$ and $7 \mu \mathrm{m}$ to simulate the nuclei and mitochondria, respectively.

Pathological studies on liver tissues have shown that the main scattering structures are cell nuclei (average diameter about $7 \mu \mathrm{m}$ ) and mitochondria (average diameter about $0.7 \mu \mathrm{m}$ ) with concentration ratio of approximately $1: 1000 .^{37,38}$ Therefore, we use a bi-component scattering model to approximate the scattering properties of the cancerous tissues. The bi-component model contains two types of spheres with 7 and $0.7 \mu \mathrm{m}$ diameters, representing the nuclei and mitochondria, respectively. Considering that the cancerization process often leads to many structural damages including excessive cell proliferation and crowed organelles, we increase the concentration of both the components to simulate the influence of cancer. Figure 5 shows the MC simulated results of the relative contrasts for intensity, DP, DOP and RLPI parameter $\mathrm{B}$ at different bi-component concentrations. In the simulation, the total scattering coefficient is increased from 70 to $220 \mathrm{~cm}^{-1}$, and the ratio of scattering coefficients between large and small spheres is set to be $3: 1$ according to the concentration ratio for nuclei and mitochondria. As shown in Fig. 5, the contrasts for the four different imaging parameters satisfy the relation: RLPI parameter $\mathrm{B}>$ intensity $>\mathrm{DP}>\mathrm{DOP}$, which agrees with the experimental results shown in Fig. 3. The bi-component model is capable for the explanation of polarization imaging experiments. Moreover, both the experimental and simulated results indicate that the RLPI parameter B is a potential indicator for the cancers in isotropic tissues. It should be pointed out that for cancerous liver tissues the bicomponent system is a simplified model. However, the real biological tissues are complex, their structural parameters are more elaborate. Therefore, for a better fit between the experiments and $\mathrm{MC}$ simulations, a multi-component model may be more suitable for future quantitative studies.

\section{Conclusion}

In summary, in this paper we applied RLPI, DPI and DOPI to cancerous liver tissues, and compared relative contrasts among these techniques. The experimental results show that both the healthy and cancerous liver tissues are highly isotropic, and the RLPI parameter B provides a good imaging contrast of cancerous liver tissues. For a better understanding of the contrast mechanism for the polarization imaging of cancerous liver tissues, we proposed a bi-component scattering model and carried on MC simulations. The scattering model consists of two types of spherical scatterers to simulate the nuclei and mitochondria, respectively. A good agreement was obtained between the experimental and simulated relative contrasts for the four polarization imaging parameters. It is also shown that polarization imaging techniques are potentially powerful tools for detection of cancerous tissues.

\section{Acknowledgments}

This work was supported by the National Natural Science Foundation of China (NSFC) Grants No. 10974114, 11174178, 41106034 and Open Fund of Key Laboratory of Optoelectronic Information and Sensing Technologies of Guangdong Higher Education Institutes, Jinan University.

\section{References}

1. V. Backman, M. B. Wallace, L. T. Perelman, J. T. Arendt, R. Gurjar, M. G. Muller, Q. Zhang, G. Zonios, E. Kline, T. McGillican, S. Shapshay, T. Valdez, K. Badizadegan, J. M. Crawford, M. Fitzmaurice, S. Kabani, H. S. Levin, M. Seiler, R. R. Dasari, I. Itzkan, J. Van Dam, M. S. Feld, "Detection of preinvasive cancer cells," Nature 406(6791), $35-36$ (2000). 
2. V. V. Tuchin, L. V. Wang, D. A. Zimnyakov, Tissue Structure and Optical Models, E. Greenbaum ed., Springer, Berlin Heidelberg, New York (2006).

3. S. K. Gayen, R. R. Alfano. "Emerging optical biomedical imaging techniques," Opt. Photonics News 7(3), 17-22 (1996).

4. A. Pierangelo, A. Nazac, A. Benali, P. Validire, H. Cohen, T. Novikova, B. Haj Ibrahim, S. Manhas, C. Fallet, M. Antonelli, A. Martino, "Polarimetric imaging of uterine cervix: A case study," Opt. Express 21(12), 14120-14130 (2013).

5. A. Pierangelo, S. Manhas, A. Benali, C. Fallet, J. Totobenazara, M. Antonelli, T. Novikova, B. Gayet, A. Martino, P. Validire, "Multispectral Mueller polarimetric imaging detecting residual cancer and cancer regression after neoadjuvant treatment for colorectal carcinomas," J. Biomed. Opt. 18(4), 046014 (2013).

6. R. S. Gurjar, V. Backman, L. T. Perelman, I. Georgakoudi, K. Badizadegan, I. Itzkan, R. R. Dasari, M. S. Feld, "Imaging human epithelial properties with polarized light-scattering spectroscopy," Nat. Med. 7(11), 1245-1248 (2001).

7. L. Silvestri, A. L. Allegra Mascaro, J. Lotti, L. Sacconi, F. S. Pavone, "Advanced optical techniques to explore brain structure and function," $J$. Innov. Opt. Health Sci. 6(1), 1230002 (2013).

8. Y. A. Ushenko, O. I. Telenga, A. P. Peresunko, O. K. Numan, "New parameter for describing and analyzing the optical-anisotropic properties of biological tissues," J. Innov. Opt. Health Sci. 4(4), 463-475 (2011).

9. S. L. Jacques, J. C. Ramella-Roman, K. Lee. "Imaging skin pathology with polarized light," $J$. Biomed. Opt. 7(3), 329-340 (2002).

10. J. C. Ramella-Roman, K. Lee, S. A. Parahl, S. L. Jacques. "Design, testing, and clinical studies of a handheld polarized light camera," J. Biomed. Opt. 9(6), 1305-1310 (2004).

11. S. L. Jacques, R. Samathama, S. Isenhath, K. Lee, "Polarized light camera to guide surgical excision of skin cancers," Proc. SPIE 6842, 684201 (2008).

12. L. P. Yu, J. C. Guo, L. D. Chou, T. L. Ma, J. S. Wu, J. D. Lee, C. Chou, "Polarization-sensitive optical coherence tomography using a modified balance detector," J. Innov. Opt. Health Sci. 5(4), 1250024 (2012).

13. R. R. Anderson, "Polarized light examination and photography of the skin," Arch. Dermatol. 127(7), 1000-1005 (1991).

14. A. Pierangel, S. Manhas, A. Benali, C. Fallet, M. Antonelli, T. Novikova, B. Gayet, P. Validire, A. Martino, "Ex vivo photometric and polarimetric multilayer characterization of human healthy colon by multispectral Mueller imaging," J. Biomed. Opt. 17(6), 066009 (2012).

15. M. Antonelli, A. Pierangelo, T. Novikova, P. Validire, A. Benali, B. Gayet, A. Martino, "Mueller matrix imaging of human colon tissue for cancer diagnostics: How Monte Carlo modeling can help in the interpretation of experimental data," Opt. Express 18(10), 1582-1593 (2010).

16. N. Ghosh, I. A. Vitkin, "Tissue polarimetry: Concepts, challenges, applications, and outlook," $J$. Biomed. Opt. 16(11), 110801 (2011).

17. A. Pierangel, A. Benali, M. Antonelli, T. Novikova, P. Validire, B. Gayet, A. Martino, "Ex-vivo characterization of human colon cancer by Mueller polarimetric imaging," Opt. Express 19, 1582-1593 (2011).

18. S. Lu, R. Chipman, "Interpretation of Mueller matrices based on polar decomposition," J. Opt. Soc. Am. A 13(5), 1106-1113 (1996).

19. N. Ghosh, M. F. G. Wood, I. A. Vitkin, "Influence of the order of the constituent basis matrices on the Mueller matrix decomposition-derived polarization parameters in complex turbid media such as biological tissues," Opt. Commun. 283(6), 1200-1208 (2010).

20. N. Ghosh, M. F. G. Wood, I. A. Vitkin, "Mueller matrix decomposition for extraction of individual polarization parameters from complex turbid media exhibiting multiple scattering, optical activity, and linear birefringence," J. Biomed. Opt. 13(4), 044036 (2008).

21. N. Ghosh, M. F. G. Wood, S. Li, R. D. Weisel, B. C. Wilson, R. Li, I. A. Vitkin, "Mueller matrix decomposition for polarized light assessment of biological tissues," J. Biophotonics 2(3), 145-156 (2009).

22. P. G. Ellingsen, M. B. Lilledahl, L. M. S. Aas, C. de L. Davies, M. Kildemo, "Quantitative characterization of articular cartilage using Mueller matrix imaging and multiphoton microscopy," J. Biomed. Opt. 16(11), 116002 (2011).

23. S. Alali, K. J. Aitken, A. Shroder, D. J. Bagli, I. A. Vitkin, "Optical assessment of tissue anisotropy in ex vivo distended rat bladders," J. Biomed. Opt. 17 (8), 086010 (2012).

24. R. Liao, N. Zeng, X. Y. Jiang, D. Z. Li, T. L. Yun, Y. H. He, H. Ma, "Rotating linear polarization imaging technique for anisotropic tissues," $J$. Biomed. Opt. 15(3), 036014 (2010).

25. N. Zeng, X. Y. Jiang, Q. Gao, Y. H. He, H. Ma, "Linear polarization difference imaging and its potential applications," Appl. Opt. 48(35), 6734-6739 (2009).

26. R. Liao, N. Zeng, D. Z. Li, T. L. Yun, Y. H. He, H. $\mathrm{Ma}$, "Penetration depth of linear polarization 
imaging for two-layer anisotropic samples," Appl. Opt. 15(23), 4681-4687 (2011).

27. H. H. He, N. Zeng, D. Z. Li, R. Liao, H. Ma, "Quantitative Mueller matrix polarimetry techniques for biological tissues," J. Innov. Opt. Health Sci. 5(3), 1250017 (2012).

28. S. Demos, H. Radousky, R. Alfano, "Deep subsurface imaging in tissues using spectral and polarization filtering," Opt. Express 7(1), 23-28 (2000).

29. T. L. Yun, N. Zeng, W. Li, D. Z. Li, X. Y. Jiang, H. $\mathrm{Ma}$, "Monte Carlo simulation of polarized photon scattering in anisotropic media," Opt. Express 17 (19), 16591-16602 (2009).

30. H. H. He, N. Zeng, W. Li, T. L. Yun, R. Liao, Y. H. He, H. Ma, "Two-dimensional backscattering Mueller matrix of sphere-cylinder scattering medium," Opt. Lett. 35(14), 2323-2325 (2010).

31. H. H. He, N. Zeng, R. Liao, T. L. Yun, W. Li, Y. H. He, H. Ma, "Application of sphere-cylinder scattering model to skeletal muscle," Opt. Express 18(14), 15104-15112 (2010).

32. E. Du, H. H. He, N. Zeng, Y. H. Guo, R. Liao, Y. H. He, H. Ma, "Two-dimensional backscattering Mueller matrix of sphere-cylinder birefringence media," J. Biomed. Opt. 17(12), 126016 (2012).
33. C. F. Bohren, D. R. Huffman, Absorption and Scattering of Light by Small Particles, John Wiley and Sons, New York (1983).

34. P. L. Appleton, A. J. Quyn, S. Swift, I. Näthke, "Preparation of wholemount mouse intestine for high-resolution three-dimensional imaging using two-photon microscopy," J. Microscopy 234(2), 196-204 (2009).

35. R. L. Jamison, J. H. Donohue, D. M. Nagorney, C. B. Rosen, W. S. Harmsen, D. M. Ilstrup, "Hepatic resection for metastatic colorectal cancer results in cure for some patients," Arch. Surg. 132(5), 505-507 (1997).

36. G. Alexandrakis, F. R. Rannou, A. F. Chatziioannou, "Tomographic bioluminescence imaging by use of a combined optical-PET (OPET) system: A computer simulation feasibility study," Phys. Med. Biol. 50(17), 4225 (2005).

37. R. S. Chapkin, L. D. Davidson, L. A. Davidson, "Phospholipid molecular species composition of mouse liver nuclei. Influence of dietary n-3 fatty acid ethyl esters," Biochem. J. 287(Pt 1), 237 (1992).

38. J. Beuthan, O. Minet, J. Helfmann, M. Herrig, G. Müller, "The spatial variation of the refractive index in biological cells," Phys. Med. Biol. 41(3), 369 (1996). 